\title{
Kinetic Simulations of Nonrelativistic Perpendicular Shocks of Young Supernova Remnants. I. Electron Shock-surfing Acceleration
}

\author{
Artem Bohdan $^{1}$ (D), Jacek Niemiec ${ }^{2}$ (D), Martin Pohl ${ }^{1,3}$ (D), Yosuke Matsumoto ${ }^{4}$, Takanobu Amano ${ }^{5}$, and Masahiro Hoshino ${ }^{5}$ (DD \\ ${ }_{1}^{1}$ DESY, D-15738 Zeuthen, Germany; artem.bohdan@desy.de \\ ${ }^{2}$ Institute of Nuclear Physics Polish Academy of Sciences, PL-31342 Krakow, Poland \\ ${ }^{3}$ Institute of Physics and Astronomy, University of Potsdam, D-14476 Potsdam, Germany \\ ${ }^{4}$ Department of Physics, Chiba University, 1-33 Yayoi-cho, Inage-ku, Chiba 263-8522, Japan \\ ${ }^{5}$ Department of Earth and Planetary Science, the University of Tokyo, 7-3-1 Hongo, Bunkyo-ku, Tokyo 113-0033, Japan \\ Received 2019 March 25; revised 2019 April 20; accepted 2019 April 21; published 2019 June 6
}

\begin{abstract}
Electron injection at high Mach number nonrelativistic perpendicular shocks is studied here for parameters that are applicable to young SNR shocks. Using high-resolution large-scale two-dimensional fully kinetic particle-in-cell simulations and tracing individual particles, we in detail analyze the shock-surfing acceleration (SSA) of electrons at the leading edge of the shock foot. The central question is to what degree the process can be captured in $2 \mathrm{D} 3 \mathrm{~V}$ simulations. We find that the energy gain in SSA always arises from the electrostatic field of a Buneman wave. Electron energization is more efficient in the out-of-plane orientation of the large-scale magnetic field because both the phase speed and the amplitude of the waves are higher than for the in-plane scenario. Also, a larger number of electrons is trapped by the waves compared to the in-plane configuration. We conclude that significant modifications of the simulation parameters are needed to reach the same level of SSA efficiency as in simulations with out-of-plane magnetic field or 3D simulations.
\end{abstract}

Key words: acceleration of particles - instabilities - ISM: supernova remnants - methods: numerical - plasmas shock waves

\section{Introduction}

The current cosmic-ray (CR) origin paradigm assumes that most of the galactic $\mathrm{CR}$ population is produced at nonrelativistic forward shocks of supernova remnants (SNRs). The main acceleration mechanism considered at shocks is diffusive shock acceleration (DSA), a first-order Fermi process (e.g., Axford et al. 1977; Drury 1983; Blandford \& Eichler 1987). Astronomical observations give strong support to this paradigm. In particular, detection of broadband nonthermal emission from SNRs, extending in some objects to TeV-range gamma-rays, proves the presence of ultrarelativistic particles in these sources, though for most SNRs it is still unclear which parent particle populations (protons or electrons) generate dominant highenergy emission (Aharonian 2013).

Acceleration of particles through DSA comes from multiple interactions with the shock front, while they bounce between the shock upstream and downstream plasmas. Particle confinement to the shock vicinity is provided by elastic scattering off magnetohydrodynamic (MHD) turbulence that renders diffusive particle motions. The critical ingredient and the main unsolved problem in the DSA theory is the particle injection. CRs undergoing DSA have Larmor radii much larger than the internal shock transition width, which is commensurate with the gyroradius of the incoming protons (with shock speed $v_{\mathrm{sh}}$ ). CRs thus see the shock as a sharp discontinuity in the plasma flow. To be fed into the acceleration process, particles therefore need to be extracted from the thermal pool and pre-accelerated. Since protons have a larger initial momentum and can be easily scattered either by MHD waves embedded in the ambient plasma or by self-generated turbulence, their injection is relatively easy to account for. The problem is more severe for electrons because of their smaller mass and consequently smaller gyroradii and inertial lengths compared to protons, and is known as the electron injection problem.
Here we study electron injection at young SNR shock waves using particle-in-cell (PIC) numerical simulations that provide a fully self-consistent treatment of the electron scales. Such shocks are characterized by high sonic, $M_{\mathrm{s}}$, and Alfvénic, $M_{\mathrm{A}}$, Mach numbers. Present observational data do not give clear constraints on the large-scale magnetic-field configuration in portions of SNR shocks from which strong nonthermal emission is detected. Radio polarimetry is notoriously difficult to interpret (e.g., Stroman \& Pohl 2009). Different approaches to data modeling for the same source can suggest the presence of quasiperpendicular fields (Petruk et al. 2009; Schneiter et al. 2010; West et al. 2016) or the opposite, quasi-parallel configurations (Rothenflug et al. 2004; Bocchino et al. 2011; Schneiter et al. 2015). As in our recent studies (Matsumoto et al. 2012, 2013, 2015; Wieland et al. 2016; Bohdan et al. 2017), in this work we examine perpendicular shocks as the most simple form of a quasi-perpendicular magnetic-field configuration. The physics of such shocks is governed by the reflection of ions at the shock caused by shock potential (Figure 1), the interaction of which with the incoming plasma excites a variety of instabilities upstream from the shock. The most important instabilities in the regime of high Mach numbers are the electrostatic two-stream Buneman instability at the leading edge of the foot, resulting from the interaction between cold incoming electrons and reflected ions (Buneman 1958), and the Weibel instability in the shock foot driven by the interaction of the incoming and reflected ions (Kato \& Takabe 2010; Niemiec et al. 2012; Matsumoto et al. 2015; Wieland et al. 2016).

The Buneman instability can mediate the generation of suprathermal electrons via shock-surfing acceleration (SSA). In a 1D picture the Buneman instability produces strong, coherent electrostatic waves that capture electrons and let them be accelerated by the convective electric field (Hoshino \& Shimada 2002), thus providing efficient electron injection. A 


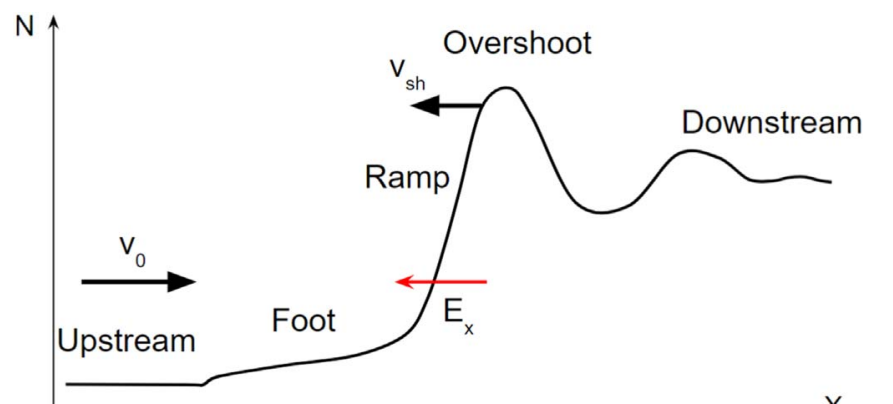
$X$

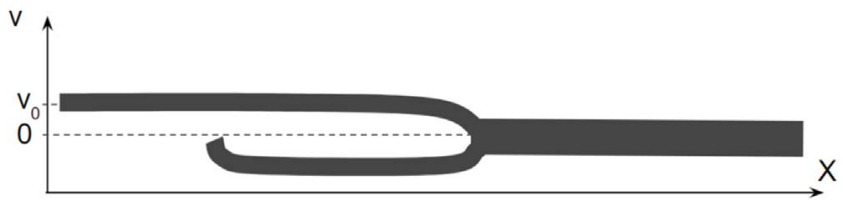

Figure 1. Perpendicular shock structure. The top panel is the particle number density profile. The shock transition consists of a foot, a ramp, an overshoot, and the downstream region. $E_{x}$ is the shock potential. $v_{0}$ and $v_{\mathrm{sh}}$ are the upstream and the shock velocities. The bottom panel is the $x$-component of the ion phase-space distribution.

number of 2D simulations of perpendicular shocks (Amano \& Hoshino 2009a; Matsumoto et al. 2012, 2013; Wieland et al. 2016) demonstrated that the length of the potential wells is limited to about the ion inertial length. Electrons can thus escape from the trapping region and re-enter it from the downstream or the upstream side to experience multiple surfingacceleration events (Amano \& Hoshino 2009a; Matsumoto et al. 2012).

The Weibel instability generates strong magnetic fields with filamentary structure. It was also recently shown with 2D simulations that spontaneous turbulent magnetic reconnection in the Weibel instability region can lead to electron acceleration (Matsumoto et al. 2015). Thin current sheets (magnetic filaments) become unstable and break up into chains of magnetic islands and X-points. Particles can be accelerated while interacting with these structures.

The spectrum of waves generated at the shock is usually at least two-dimensional. Which of the unstable modes appears in a 2D simulation strongly depends on the configuration of the mean magnetic field though, as modes may be artificially suppressed if their wavevector is not contained in the simulation plane. In Bohdan et al. (2017) we showed that the Weibel instability is best reproduced with the in-plane setup, whereas the Buneman modes are considerably stronger and more coherent with a strictly out-of-plane orientation. Suprathermal tails in the electron spectra are found for all simulated shocks, and the initial acceleration of electrons always occurs through the SSA process in the Buneman wave region. However, the subsequent stages of injection strongly depend on the field configuration. For an out-of-plane field, adiabatic heating dominates the spectral evolution. For configurations with an in-plane magnetic-field component, particles are non-adiabatically accelerated in interactions with turbulent magnetic structures in the shock, resembling a second-order Fermi process, and magnetic reconnection also occurs. The fraction of nonthermal electrons is an order of magnitude larger for the out-of-plane configuration than for other field orientations, mainly on account of a higher SSA efficiency.
The first 3D PIC simulation of a high- $M_{\mathrm{A}}$ shock was presented by Matsumoto et al. (2017) for an oblique subluminal configuration, $c / \tan \Theta_{\mathrm{Bn}}>v_{\mathrm{sh}}$, where $\Theta_{\mathrm{Bn}}$ is the angle of the large-scale magnetic field with respect to the shock normal, $v_{\mathrm{sh}}$ is the shock velocity, and $c$ is the speed of light. Buneman waves and Weibel magnetic turbulence were found to coexist in the shock structure. Energetic electrons that initially experienced SSA underwent pitch-angle diffusion by interacting with magnetic turbulence in the shock foot and ramp. This provides confinement in the shock transition region during which particles gain energy by shock drift acceleration (SDA). The computational cost of 3D experiments is still too high to sample the range of plasma conditions that one may find in SNR shocks. Nevertheless, the 3D results indicate which parts of 3D shock physics can be reliably probed with 2D simulations.

In this work we report on new large-scale 2D fully kinetic PIC simulations of nonrelativistic strictly perpendicular shocks in the regime of high Mach numbers, $M_{\mathrm{A}} \gtrsim 20$ and $M_{\mathrm{s}} \gtrsim 30$, as appropriate for forward shocks of young SNRs. The simulations are conducted in a $2 \mathrm{D} 3 \mathrm{~V}$ configuration, i.e., we follow two spatial coordinates and all three components of the velocity and the electromagnetic fields. Numerical experiments are performed for both in-plane and out-of-plane configurations of the large-scale magnetic field. These simulations complement our previous investigations of 2D perpendicular shocks (e.g., Matsumoto et al. 2012, 2013, 2015; Wieland et al. 2016; Bohdan et al. 2017). The aim of this work is to analyze in detail the initial energization via SSA in the Buneman-instability region. The successive acceleration in the shock foot and ramp on account of, e.g., inelastic scattering off the Weibelinstability turbulence, is the subject of a separate publication.

Conditions for efficient electron energization via SSA were first investigated by Matsumoto et al. (2012), supported with PIC simulations with out-of-plane magnetic-field configuration. The process occurs in low-temperature (low beta) plasmas, in which the Buneman instability can effectively grow. For efficient acceleration the electrostatic waves should also be strong enough to trap electrons and hold them during acceleration, which defines a minimum Alfvénic Mach number for a shock to be capable of producing relativistic electrons via SSA,

$$
M_{\mathrm{A}} \geqslant(1+\alpha)\left(\frac{m_{\mathrm{i}}}{m_{\mathrm{e}}}\right)^{\frac{2}{3}},
$$

where $\alpha$ is the flux ratio of reflected to incoming ions and $m_{\mathrm{i}}$ and $m_{\mathrm{e}}$ are the ion and the electron mass, respectively. In the presence of an in-plane magnetic field the motion of the reflected ions is not fully contained in the simulation grid and thus the corresponding component of the Buneman waves cannot be captured (Bohdan et al. 2017). To account for this effect we proposed a modified trapping condition:

$$
M_{\mathrm{A}} \geqslant \sqrt{\frac{2}{1+\sin ^{2} \varphi}}(1+\alpha)\left(\frac{m_{\mathrm{i}}}{m_{\mathrm{e}}}\right)^{\frac{2}{3}},
$$

where $\varphi$ is the orientation angle of the large-scale perpendicular magnetic field with respect to the simulation plane, with $\varphi=0^{\circ}$ representing the in-plane configuration (see Figure 2). The earlier 2D simulations of Bohdan et al. (2017) all satisfied the trapping condition of Equation (1) and were performed for a 


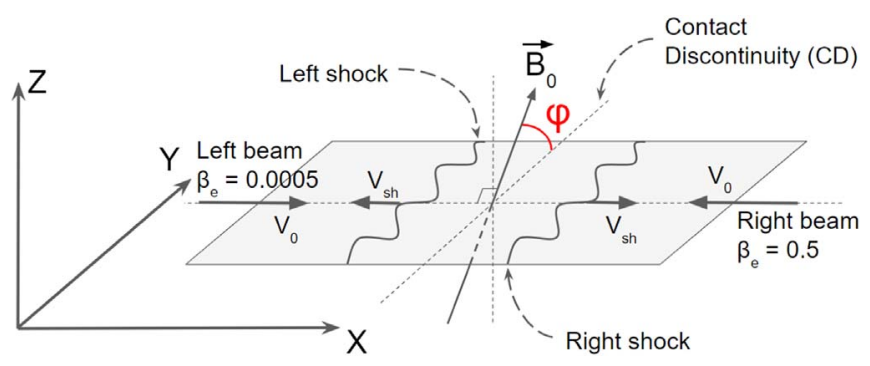

Figure 2. Illustration of the simulation setup.

single value of the reduced mass ratio, $m_{\mathrm{i}} / m_{\mathrm{e}}=100$, and a small $\left(\beta_{\mathrm{e}} \ll 1\right)$ or moderate $\left(\beta_{\mathrm{e}}=0.5\right)$ plasma beta. Our present work augments this analysis with investigations of the trapping conditions of Equations (1) and (2) and SSA efficiency for different mass ratios in the range $m_{\mathrm{i}} / m_{\mathrm{e}}=$ 50-400. Matsumoto et al. (2017) demonstrated that the SSA process is well reproduced with 2D out-of-plane simulations, but processes in the shock ramp and overshoot are suppressed. On the other hand, the stochastic Fermi-like acceleration in the Weibel-instability-generated turbulence works similarly in 2D in-plane experiments. If the modified trapping condition defines the parameter range for which we have the same efficiency of electron pre-acceleration for an in-plane configuration as that observed in 2D simulations with an out-of-plane magnetic field, it is possible to reproduce realistic 3D physics with far cheaper $2 \mathrm{D}$ experiments with $\varphi=0^{\circ}$. This is the main hypothesis under discussion here.

The paper is organized as follows. We present a description of the simulation setup in Section 2. The results are presented in Section 3. Section 4 contains the summary and discussion.

\section{Simulation Setup}

The simulation setup adopted in this work is the same as that used in Bohdan et al. (2017) and illustrated in Figure 2. As a result of the collision of two counter-streaming electron-ion plasma beams, two shocks are formed that propagate in opposite directions and are separated by a contact discontinuity (CD). The plasma flow is set along the $x$-direction in the $x y$ plane. Plasma particles are continuously injected at both sides of the simulation box with velocities $\boldsymbol{v}_{\mathrm{L}}=v_{\mathrm{L}} \hat{\boldsymbol{x}}$ and $\boldsymbol{v}_{\mathrm{R}}=v_{\mathrm{R}} \hat{\boldsymbol{x}}$, where the indices $\mathrm{L}$ and $\mathrm{R}$ refer, respectively, to the left and right sides of the simulation box. As the two shocks move away from the $\mathrm{CD}$ in the left and the right plasma, we refer to them as the left and the right shocks, respectively. The two plasma streams carry a homogeneous magnetic field, $\boldsymbol{B}_{0}$, that is perpendicular to the shock normal and lies in the $y z$ plane. The magnetic field thus forms an angle $\varphi$ with the $y$-axis. Initialized with the flow is a motional electric field $\boldsymbol{E}_{0}=-\boldsymbol{v} \times \boldsymbol{B}_{0}$, with $\boldsymbol{v}=\boldsymbol{v}_{\mathrm{L}}$ or $\boldsymbol{v}=\boldsymbol{v}_{\mathrm{R}}$, respectively, for the left and right beams. We assume that the beams move with equal absolute velocities, $v_{\mathrm{L}}=v_{\mathrm{R}}=0.2 c$, and that the magnetic-field strength in both plasmas is equal, $\boldsymbol{B}_{0 \mathrm{~L}}=\boldsymbol{B}_{0 \mathrm{R}}$. The motional electric field thus has equal strength and opposing signs in the two slabs. We use the method of Wieland et al. (2016) to suppress the artificial electromagnetic transient that results from the initial strong electric-field gradient between the two plasma slabs.
We collide plasma beams of equal density but different temperatures, thus studying two different shocks in one simulation. The temperature ratio between the two beams is 1000 , such that the sonic Mach numbers, $M_{\mathrm{s}}$, of the two shocks differ by a factor of $\sqrt{1000} \simeq 30$. In terms of the electron plasma beta (the ratio of the electron plasma pressure to the magnetic pressure) the left beam has $\beta_{\mathrm{e}, \mathrm{L}}=5 \times 10^{-4}$ and the right beam has $\beta_{\mathrm{e}, \mathrm{R}}=0.5$. This choice of plasma beta facilitates a direct comparison with our earlier work (Bohdan et al. 2017) and also with results of previous 2D simulations of perpendicular shocks (Matsumoto et al. 2012, 2013) and a 3D simulation of a quasi-perpendicular shock (Matsumoto et al. 2017 ), in which $\beta_{\mathrm{e}, \mathrm{R}}=0.5$ is assumed. Note that our system is approximately in ram-pressure balance, and consequently the simulation frame is also the downstream rest frame of the two shocks.

The parameters of the simulation runs described in this paper are listed in Table 1. We have performed seven large-scale numerical experiments (runs A-G), that feature in total 14 simulated shocks. Here we refer to each of these shock cases as a separate simulation run, and tag the shocks in the left plasma $\left(\beta_{\mathrm{e}, \mathrm{L}}=5 \times 10^{-4}\right)$ with ${ }^{*} 1$, and the right shocks with ${ }^{*} 2\left(\beta_{\mathrm{e}, \mathrm{R}}=0.5\right)$. Simulation runs A-F assume the in-plane magnetic-field configuration, $\varphi=0^{\circ}$, and run $\mathrm{G}$ uses the out-of-plane magnetic-field orientation, $\varphi=90^{\circ}$. We do not consider simulations with $\varphi=45^{\circ}$, because the shock structure and the acceleration mechanisms observed in this case are almost identical to those in runs with the in-plane field configuration (Bohdan et al. 2017). The runs with the in-plane magnetic field cover a wide range of ion-to-electron mass ratios and Alfvénic Mach numbers, as illustrated in Figure 3, which permits an investigation of the influence of these parameters on the electron acceleration efficiency and to scale our results to the realistic ion-to-electron mass ratio. Note that some aspects of the shock physics in runs B and $\mathrm{G}$ have been discussed in our previous paper (see runs A and C in Bohdan et al. 2017).

The derived shock properties are also listed in Table 1. The Alfvén velocity is defined as $v_{\mathrm{A}}=B_{0} / \sqrt{\mu_{0}\left(N_{\mathrm{e}} m_{\mathrm{e}}+N_{\mathrm{i}} m_{\mathrm{i}}\right)}$, where $\mu_{0}$ is the vacuum permeability, $N_{\mathrm{i}}$ and $N_{\mathrm{e}}$ are the ion and the electron number densities, and $B_{0}$ is the far-upstream magneticfield strength. The sound speed reads $c_{\mathrm{s}}=\left(\Gamma k_{\mathrm{B}} T_{\mathrm{i}} / m_{\mathrm{i}}\right)^{1 / 2}$, where $k_{\mathrm{B}}$ is the Boltzmann constant, $\Gamma$ is a nonrelativistic adiabatic index. The ion themperature $T_{i}$ reads $k_{B} T_{\mathrm{i}}=m_{i} \frac{v_{t h}^{2}}{2}$, where $v_{t h}$ is the most probable speed of the upstream ions in the upstream reference frame. The Alfvénic, $M_{\mathrm{A}}=v_{\mathrm{sh}} / v_{\mathrm{A}}$, and sonic, $M_{\mathrm{s}}=v_{\mathrm{sh}} / c_{\mathrm{s}}$, Mach numbers of the shocks in Table 1 are given in the conventional upstream reference frame. As the in-plane and the out-of-plane magnetic field lead to a different number of degrees of freedom, the adiabatic indices are different, with $\Gamma=5 / 3$ and $\Gamma=2$, respectively, for $\varphi=0^{\circ}$ and $\varphi=90^{\circ}$. Thus, the resulting expected shock speeds take values $v_{\mathrm{sh}}=0.263 c$ for runs $\mathrm{A}-\mathrm{F}$ and $v_{\mathrm{sh}}=0.294 c$ for runs $\mathrm{G}$. In the simulation frame the speeds are smaller by the shock compression ratio.

To investigate the role of SSA in electron pre-acceleration, we adjust the magnetic-field strength, $B_{0}$, to establish Alfvénic Mach numbers that test the trapping conditions defined by Equations (1) and (2). A comparison of the Alfvénic Mach numbers and the mass ratio of all runs with trapping limits is offered in Figure 3. Nevertheless, we always consider weakly magnetized plasmas with the ratio of the electron plasma frequency, $\omega_{\mathrm{pe}}=\sqrt{e^{2} N_{\mathrm{e}} / \epsilon_{0} m_{\mathrm{e}}}$, to the electron gyrofrequency, $\Omega_{\mathrm{e}}=e B_{0} / m_{\mathrm{e}}$, in the range $\omega_{\mathrm{pe}} / \Omega_{\mathrm{e}}=8.5-17.3$. Here, $e$ is the 
Table 1

Simulation Parameters

\begin{tabular}{|c|c|c|c|c|c|c|c|c|c|c|c|c|}
\hline \multirow{2}{*}{ Runs } & \multirow{2}{*}{$\varphi$} & \multirow{2}{*}{$L_{y}\left(\lambda_{\mathrm{si}}\right)$} & \multirow{2}{*}{$m_{\mathrm{i}} / m_{\mathrm{e}}$} & \multirow{2}{*}{$\omega_{\mathrm{pe}} / \Omega_{\mathrm{e}}$} & \multirow{2}{*}{$M_{\mathrm{A}}$} & \multicolumn{2}{|c|}{$M_{\mathrm{s}}$} & \multicolumn{2}{|c|}{$\beta_{\mathrm{e}}$} & \multirow{2}{*}{$\begin{array}{c}\text { Equation (1) } \\
\alpha=0.2\end{array}$} & \multicolumn{2}{|c|}{ Equation (2) } \\
\hline & & & & & & ${ }^{*} 1$ & ${ }^{*} 2$ & *1 & ${ }^{*} 2$ & & $\overline{\alpha=0.2}$ & $(0.5)$ \\
\hline$\overline{\mathrm{A} 1, \mathrm{~A} 2}$ & $0^{\circ}$ & 10.9 & 50 & 12 & 22.6 & 1104 & 35 & $5 \times 10^{-4}$ & 0.5 & 16 & 22.4 & $\overline{(28)}$ \\
\hline $\mathrm{C} 1, \mathrm{C} 2$ & $0^{\circ}$ & 12 & 100 & 17.3 & 46 & 2242 & 71 & $5 \times 10^{-4}$ & 0.5 & 26 & 36 & (46) \\
\hline D1, D2 & $0^{\circ}$ & 11.9 & 200 & 8.5 & 32 & 1550 & 49 & $5 \times 10^{-4}$ & 0.5 & 41 & 58 & (72) \\
\hline E1, E2 & $0^{\circ}$ & 11.9 & 200 & 12 & 44.9 & 2191 & 69 & $5 \times 10^{-4}$ & 0.5 & 41 & 58 & (72) \\
\hline $\mathrm{F} 1, \mathrm{~F} 2$ & $0^{\circ}$ & 8.2 & 400 & 12 & 68.7 & 3353 & 106 & $5 \times 10^{-4}$ & 0.5 & 65 & 92 & (115) \\
\hline
\end{tabular}

Note. Parameters of simulation runs described in this paper. Listed are: the orientation of the uniform perpendicular magnetic field with respect to the $2 \mathrm{D}$ simulation plane, $\varphi$, the transverse size of the computational box, $L_{y}$, in units of the ion skin depth, $\lambda_{\mathrm{si}}$, the ion-to-electron mass ratio $m_{\mathrm{i}} / m_{\mathrm{e}}$, the plasma magnetization, $\omega_{\mathrm{pe}} / \Omega_{\mathrm{e}}$, and Alfvénic and sonic Mach numbers, $M_{\mathrm{A}}$ and $M_{\mathrm{s}}$, the latter separately for the left (runs ${ }^{*} 1$ ) and the right (runs ${ }^{*} 2$ ) shock. We also list the electron plasma beta, $\beta_{\mathrm{e}}$, for each simulated shock and the critical Alfvénic Mach number (Equation (1)) for $\alpha=0.2$, as well as the modified trapping condition (Equation (2)) calculated for $\alpha=0.2$ and $\alpha=0.5$ (in brackets). All runs use the electron skin depth of $\lambda_{\mathrm{se}}=20 \Delta$.

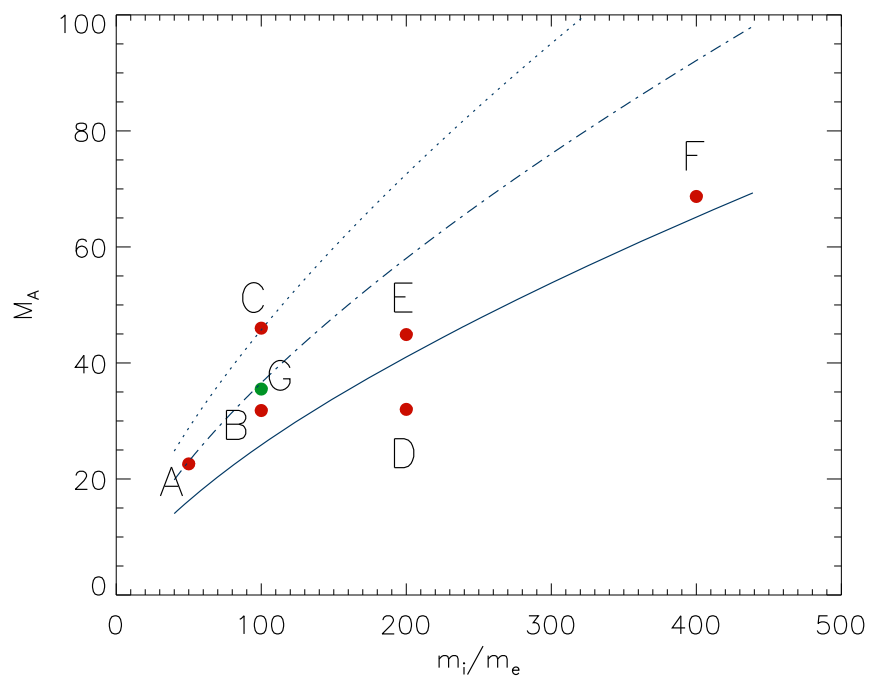

Figure 3. Alfvénic Mach numbers and mass ratios of the simulation runs. Runs A-F with an in-plane magnetic-field configuration are depicted with red dots. Run $\mathrm{G}$ with an out-of-plane field is marked with a green dot. The blue solid line shows the scaling given by the trapping condition of Equation (1), calculated for $\alpha=0.2$. The blue dashed-dotted and dotted lines show the modified trapping conditions (Equation (2)) for $\alpha=0.2$ and $\alpha=0.5$, respectively.

electron charge, and $\epsilon_{0}$ is the vacuum permittivity. To keep the plasma beta constant we adjust the plasma temperatures and hence the sound speeds and resulting sonic Mach numbers (see Table 1).

In this work we want to verify several hypotheses. The first is the scaling of the SSA efficiency with the ion-to-electron mass ratio for shocks that fulfill the trapping condition of Equation (1), here applied to the in-plane magnetic-field configurations. Runs $\mathrm{A}, \mathrm{B}, \mathrm{E}$, and $\mathrm{F}$ define the set of simulations conducted for $m_{\mathrm{i}} / m_{\mathrm{e}}=50,100,200$, and 400, respectively.

The second objective is the modified trapping condition of Equation (2). We test this condition by conducting simulation runs $\mathrm{C}$, which satisfy Equation (2) for $\alpha \leqslant 0.5$. The question to be addressed is whether 2D simulations with an in-plane magnetic-field configuration can reproduce the SSA efficiency observed in 2D runs with the same $m_{\mathrm{i}} / m_{\mathrm{e}}$ and the out-of-plane fields, here marked as runs $\mathrm{G}$.
The third set of simulations consists of runs $\mathrm{D}$ and $\mathrm{E}$, performed for the same mass ratio $m_{\mathrm{i}} / m_{\mathrm{e}}=200$. The Alfvénic Mach number in run D clearly violates Equation (1), so we expect a very low intensity of Buneman waves. Nevertheless, particle acceleration can still occur in the shock foot and ramp, whose structure is defined by the magnetic filaments, and we are interested in the nonthermal electron population that forms in the absence of SSA. Note that cross-comparison of runs B and $\mathrm{D}$, and $\mathrm{C}$ and $\mathrm{E}$ can yield the mass-ratio dependence for shocks with the same Alfvénic Mach numbers.

The electron skin depth in the upstream plasma is common for all runs and equals $\lambda_{\mathrm{se}}=20 \Delta$, where $\Delta$ is the size of grid cells. The ion skin depth, $\lambda_{\mathrm{si}}=\sqrt{m_{\mathrm{i}} / m_{\mathrm{e}}} \lambda_{\mathrm{se}}$, is used here as the unit of length. The timescale and all temporal dependencies are given in terms of the upstream ion Larmor frequency, $\Omega_{\mathrm{i}}$, where $\Omega_{\mathrm{i}}=e B_{0} / m_{\mathrm{i}}$. The simulation time is typically $t=(6-8) \Omega_{\mathrm{i}}^{-1}$, which is enough to cover at least a few shock self-reformation cycles (see Bohdan et al. 2017). The time step we use is $\delta t=1 / 40 \omega_{\mathrm{pe}}^{-1}$.

The two plasma beams injected at sides of the simulation box are composed of an equal number of ions and electrons, $N_{\text {ppc }}=20$. Electron and ion plasma pairs are initialized at the same locations to ensure the initial charge-neutrality of the system. There is no escape of particles from the computational box, and we use injection layers receding from the $\mathrm{CD}$ as in Bohdan et al. (2017), which helps alleviate numerical gridCerenkov effects and saves computational resources. The simulation box expands in the $x$-direction during the run. The final size of a simulation box can reach $L_{x} \approx 280 \lambda_{\mathrm{si}}$. The transverse size of the simulation box, $L_{y}=(8.2-24) \lambda_{\mathrm{si}}$, is large enough to cover several of the magnetic filaments, which are typically separated by $\sim \lambda_{\mathrm{si}}$, and at the same time limits the computational expense that grows quadratically with $m_{\mathrm{i}} / m_{\mathrm{e}}$. The largest simulation box of size $L_{x} \times L_{y}=(3264 \times$ $96000) \Delta$ is used in run $\mathrm{F}$ with $m_{\mathrm{i}} / m_{\mathrm{e}}=400$. Open boundary conditions are imposed in the $x$-direction and periodic boundaries are applied in the $y$-direction.

The numerical code we use is a 2D3V-adapted and modified version of the relativistic electromagnetic PIC code TRISTAN (Buneman 1993) with MPI-based parallelization (Niemiec et al. 2008; Wieland et al. 2016) and the option to trace individual particles. 


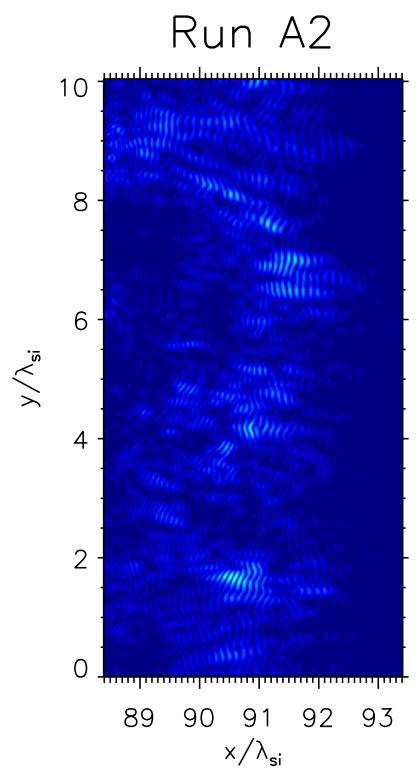

Run G2

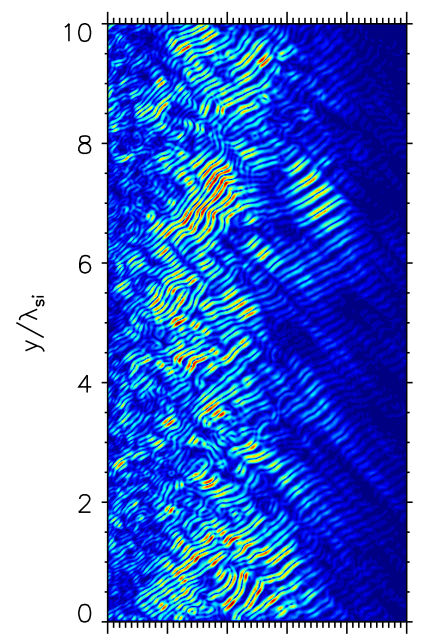

203204205206207208

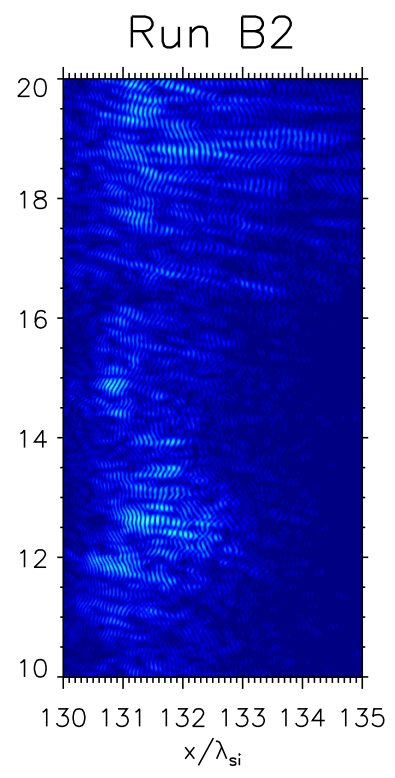

Run G2*

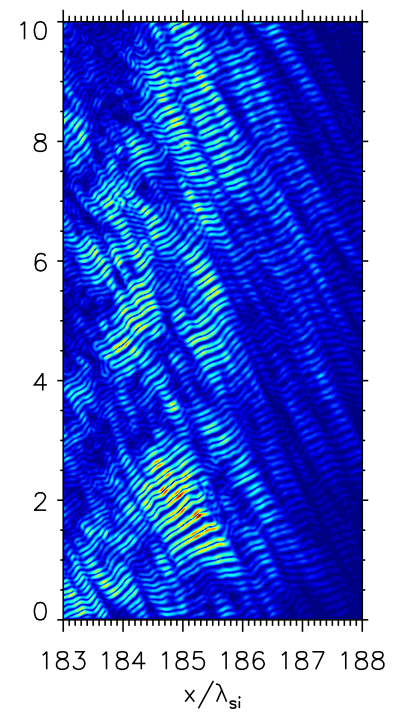

Run E2

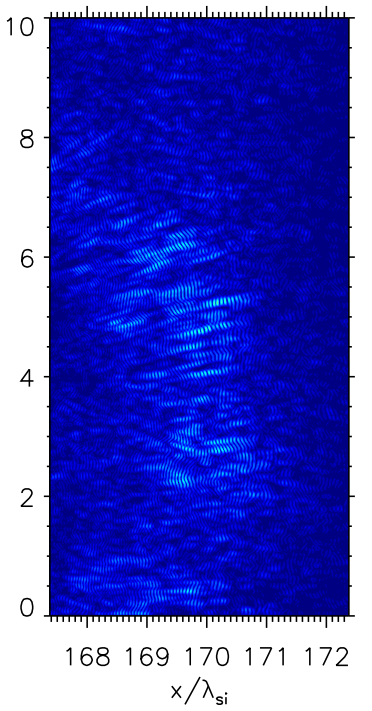

Run C2

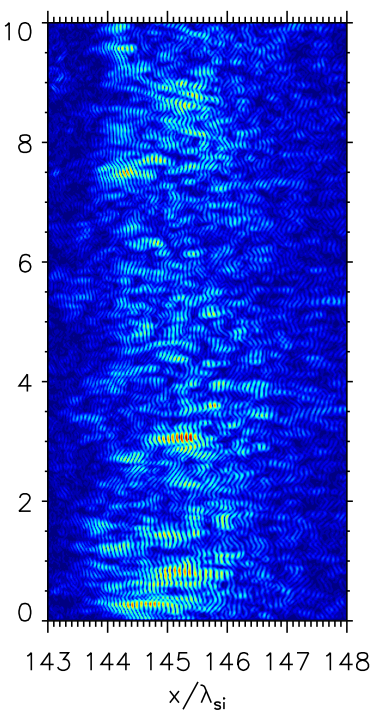

Run F2
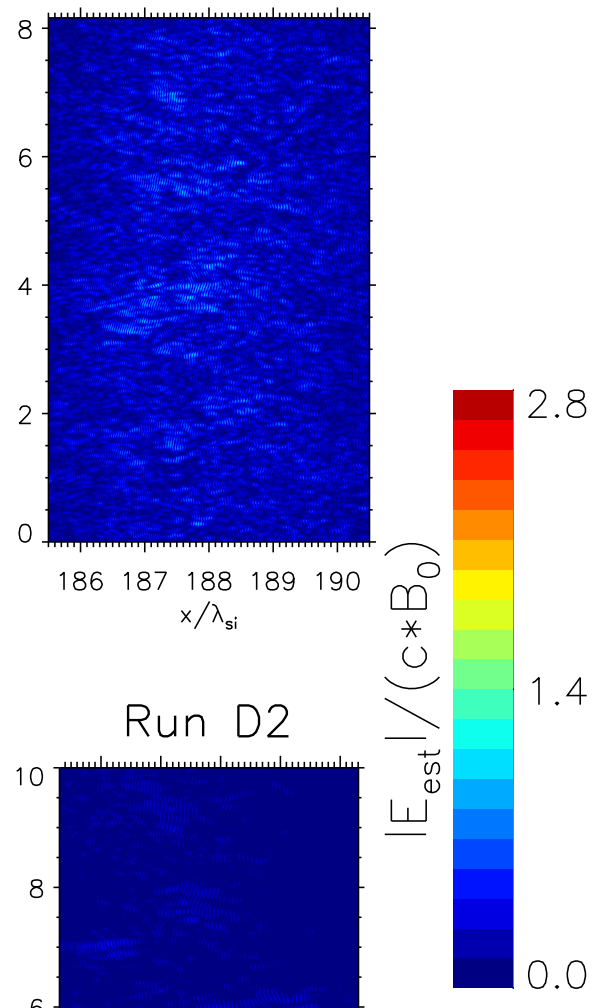

6

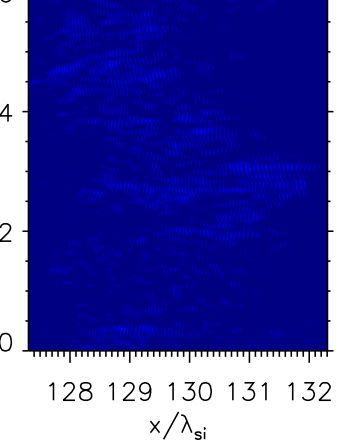

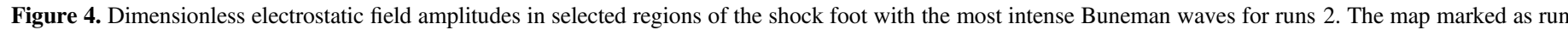
$\mathrm{G} 2 *$ is chosen at a moment when the average field strength is the same as in run $\mathrm{C} 2$.

\section{Results}

In Section 3.1 we describe the structure of the Buneman wave modes in all simulations and also summarize the findings of Bohdan et al. (2017). Then we discuss the electron acceleration efficiency through SSA in Section 3.2.

\subsection{The Buneman Instability}

Figure 4 presents the maps of the electrostatic field amplitude in the foot of the right shocks (runs A2-G2, see Table 1), which propagate in moderate-temperature plasmas with $\beta_{\mathrm{e}}=0.5$. Only portions of the simulation boxes are shown to facilitate one-to-one comparison between the runs. Run $\mathrm{G}^{*}$ is run $\mathrm{G} 2$ at a different phase of shock reformation. The electrostatic fields are calculated as $\left|E_{\mathrm{ES}}\right|=|-\nabla \phi|$, where $\phi$ is the electric potential, which is derived directly from the charge distribution. The maps are plotted for simulation times, at which the cyclic shock self-reformation allows the strongest Buneman modes. Note, that the maps for runs B2 and G2 can be compared with Figures 6(a3) and (c3), respectively, in Bohdan et al. (2017), in which results for runs B1 and G1 are presented (marked as runs $\mathrm{A} 1$ and $\mathrm{C} 1$, respectively).

The properties of the Buneman instability discussed in Bohdan et al. (2017) can be readily observed in Figure 4. The wavevectors are approximately parallel to the shock normal for the in-plane configurations (runs A2-F2) and oblique for the out-of-plane magnetic field (run G2). This reflects the motion of shock-reflected ions: for $\varphi=0^{\circ}$ the ions are confined to the $x z$-plane, whereas for $\varphi=90^{\circ}$ they stream in the simulation plane. The Buneman wave region shows a patchy structure for the in-plane field configurations that can be linked to clumps in the overshoot produced by merging magnetic filaments. In total, the Buneman waves occupy a much smaller region than the out-of-plane configuration, for which the waves are coherent and more intense.

The phase velocity of the Buneman modes matches the relative speed between shock-reflected ions and incoming electrons of the upstream plasma. Since for $\varphi=0^{\circ}$ part of the 
Table 2

Dimensionless Peak Amplitude of Buneman Waves and Fraction of Pre-accelerated Electrons

\begin{tabular}{lcc}
\hline \hline Run & $\max \left(\left|E_{\mathrm{ES}}\right| /\left(c B_{0}\right)\right)$ & $N_{\mathrm{e}, \mathrm{BI}} / N_{\mathrm{e}, \text { tot }}(\%)$ \\
\hline $\mathrm{A} 2$ & 1.1 & 0.43 \\
$\mathrm{~B} 2$ & 1.3 & 0.46 \\
$\mathrm{C} 2$ & 2.3 & 0.6 \\
$\mathrm{D} 2$ & 0.4 & 0.34 \\
$\mathrm{E} 2$ & 1.3 & 0.49 \\
$\mathrm{~F} 2$ & 1.1 & 0.44 \\
$\mathrm{G} 2$ & 2.7 & 6.8 \\
$\mathrm{G} 2 *$ & 2.3 & 2.7 \\
\hline
\end{tabular}

Note. For $\beta_{\mathrm{e}}=0.5$ shocks we list the normalized peak amplitude, $\max \left(\left|E_{\mathrm{ES}}\right| /\left(c B_{0}\right)\right)$, of the electrostatic waves, calculated as mean $\left|E_{\mathrm{ES}}\right| /\left(c B_{0}\right)$ for the 100 simulation cells with the highest $\left|E_{\mathrm{ES}}\right| /\left(c B_{0}\right)$, and the fraction of electrons pre-accelerated to $(\gamma-1)>0.1$.

ion motion is outside of the simulation grid, the wavelengths of the Buneman waves are smaller $\left(\lambda \approx 1.9 \lambda_{\mathrm{se}}\right)$ than those for the out-of-plane field, for which $\lambda \approx 3.3 \lambda_{\text {se }}$. Note, that Figure 4 shows $|E|$ and hence the wavelength is twice the separation of wavefronts, here provided in units of the ion skin depth. The surface area of the Buneman wave region for shocks in moderate-temperature plasma is $20 \%-30 \%$ larger than at the corresponding low- $\beta$ shocks, but the intensity of the waves is 20\%-50\% smaller (compare Figure 6 in Bohdan et al. 2017).

For the high- $\beta$ systems presented in Figure 4, Table 2 lists the peak amplitude of Buneman waves and the fraction of preaccelerated electrons. The runs A2, B2, E2, and F2 satisfy the trapping condition of Equation (1) (see Figure 3), and both the peak and average strength of the electrostatic field are similar. Small differences between them arise from shock reformation. We conclude that irrespective of the mass ratio, the physical conditions at shocks with $M_{\mathrm{A}}$ satisfying Equation (1) are similar. However, the electrostatic force is weaker on average than the Lorentz force on a $\gamma \gtrsim 2$ electron $\left(\left|E_{\mathrm{ES}}\right| /\left(c B_{0}\right)<1\right)$.

Considerably larger electrostatic field amplitudes, reaching $\left|E_{\mathrm{ES}}\right| /\left(c B_{0}\right) \sim 2.3$, can be observed for run $\mathrm{C} 2$. Here, the Alfvén Mach number of the shock, $M_{\mathrm{A}}=46$, is much larger than the minimum $M_{\mathrm{A}}$ defined by Equation (1) and also satisfies the modified trapping condition of Equation (2), which for the measured $\alpha \simeq 0.32$ gives the minimum $M_{\mathrm{A}} \simeq 40.2$. The field intensity in run $\mathrm{C} 2$ is about a factor of 2 larger than that in both run $\mathrm{B} 2$ with the same mass ratio, $m_{\mathrm{i}} / m_{\mathrm{e}}=100$ and run E2 with mass ratio $m_{\mathrm{i}} / m_{\mathrm{e}}=200$ but similar Alfvén Mach number, $M_{\mathrm{A}} \simeq 45$. This shows that the strength of the electrostatic modes is driven by the value of the Alfvénic Mach number in relation to the trapping condition (Equation (1)). The absolute value of $M_{\mathrm{A}}$ is not important, as in run D2 we see Buneman waves with amplitudes a factor of 3 lower than those in run B2 with the same Alfvénic Mach number. Essentially, all observed wave intensities are slightly weaker than the saturation level estimated by Ishihara et al. (1980).

The modified trapping condition (Equation (2)) was expected to compensate for the effect of the field configuration. Shocks with sufficiently large $M_{\mathrm{A}}$ should then reproduce similar Buneman wave intensities in 2D in-plane magnetic-field configurations than in simulations with the out-of-plane fields. However, the electrostatic field in run C2 is weaker by $20 \%$ than that in run $\mathrm{G} 2$ with $\varphi=90^{\circ}$. At a different phase of shock

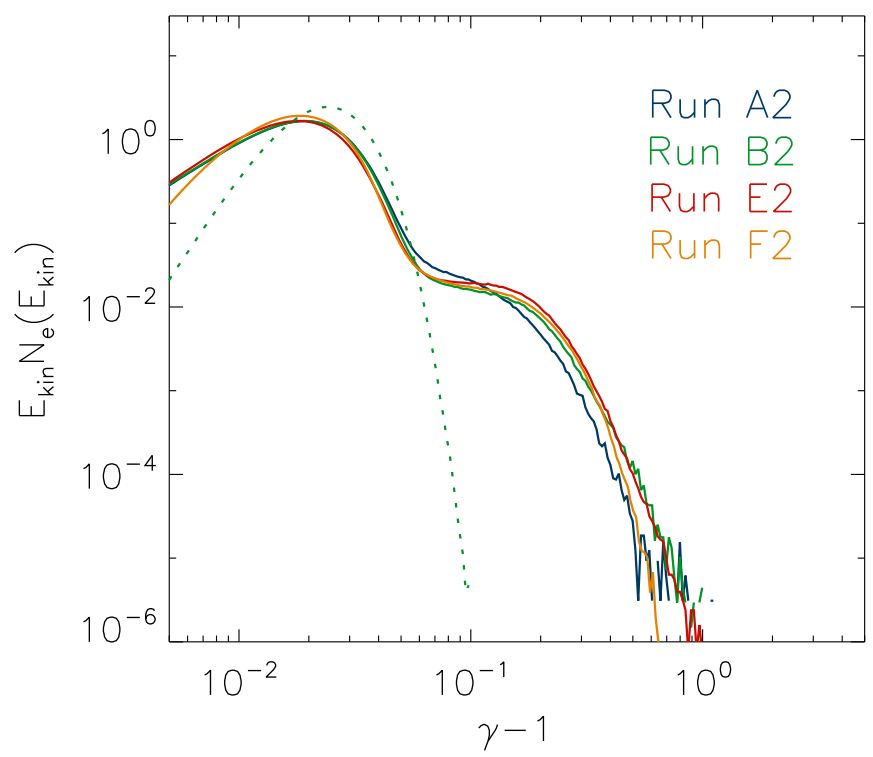

Figure 5. Simulation-frame kinetic-energy spectra of electrons in the regions of the shock foot selected for Figure 4 color-coded for run A2 (blue), run B2 (green), run E2 (red), and for run F2 (orange). The dotted green line indicates the spectrum of upstream cold plasma electrons (extracted from run B2).

reformation, run $\mathrm{G} 2$, now called $\mathrm{G} 2 *$, has the same electricfield amplitude as $\mathrm{C} 2$, but four times the number of preaccelerated electrons. This discrepancy might arise from Equation (2) only compensating for the neglect of the $z$-motion of ions. In out-of-plane simulation we observe that the relative speed between electrons and reflected ions can reach $\sim 0.6 c$, because of acceleration in upstream electric field, which is a factor of $\sim 1.5$ larger then the value assumed in the derivation of the trapping condition (see Matsumoto et al. 2012), while in in-plane case the acceleration is in $z$-direction. It may be that we need to also account for this effect by adding a factor of 1.5 to the modified trapping condition,

$$
M_{\mathrm{A}} \geqslant 1.5 \sqrt{\frac{2}{1+\sin ^{2} \varphi}}(1+\alpha)\left(\frac{m_{\mathrm{i}}}{m_{\mathrm{e}}}\right)^{\frac{2}{3}} .
$$

This equation gives $M_{\mathrm{A}} \simeq 60.3$ for the minimum Alfvén Mach number, with which the amplitudes of the Buneman waves observed at shocks with $M_{\mathrm{A}}=35.5$ in 2D simulations with outof-plane magnetic fields could be reproduced in runs applying $\varphi=0^{\circ}$ field configuration. This value is much larger than any of the Mach numbers studied here for $m_{\mathrm{i}} / m_{\mathrm{e}}=100$, and thus requires attention in the future.

\subsection{Electron Acceleration in the Buneman Zone}

Table 2 lists the fraction of electrons that have been preaccelerated in the Buneman wave zone to $(\gamma-1)>0.1$, $N_{\mathrm{e}, \mathrm{BI}} / N_{\mathrm{e}, \text { tot }}$. This fraction is much larger in run G2 than it is in runs A2-F2. Bohdan et al. (2017) argued that at least part of this difference is due to differences in the amplitude of the electrostatic waves and their coverage area.

Figures 5 and 6 show kinetic-energy spectra of electrons occupying the Buneman wave regions highlighted in Figure 4. Figure 5 shows energy spectra for runs A2, B2, E2, and F2, for which the Alfvénic Mach numbers exceed the trapping condition (Equation (1)) by a similar margin. The spectra are statistically indistinguishable, and the fraction of pre-accelerated electrons is 


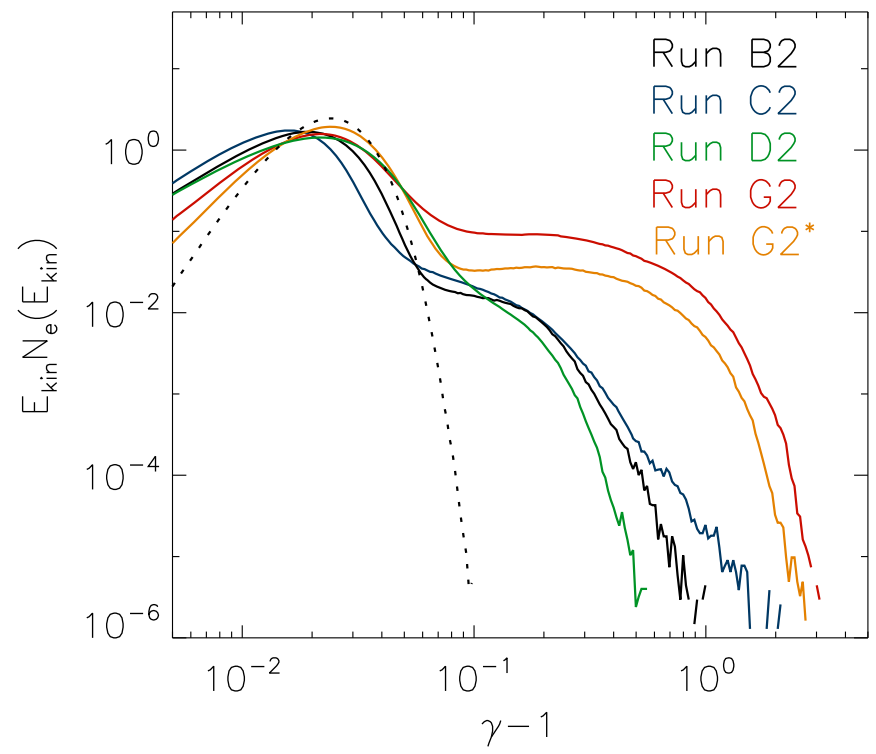

Figure 6. Spectra of electrons as in Figure 5 for run B2 (black), run C2 (blue), run D2 (green), run G2 (red), and run G2* (orange). The dotted black line indicates the spectrum of upstream cold plasma electrons (extracted from run B2).

$\sim 0.45 \%$ for all runs. This is again in line with the ion-to-electron mass-ratio dependence of the trapping condition.

Electron spectra for runs B2, C2, and D2, that probe different physical conditions at shocks with an in-plane magnetic-field configuration, are compared to the spectrum for the out-ofplane case $\mathrm{G} 2$ in Figure 6. The fractions of pre-accelerated electrons differ between the in-plane runs (see Table 2), reflecting the different intensities of the Buneman waves. In run $\mathrm{C} 2$, the spectrum extends to higher energies and contains more energetic electrons than that for run B2, which arises from the difference in Mach number. The Alfvénic Mach number of the shock in run D2 instead violates the trapping condition, and only $\sim 0.34 \%$ of electrons are pre-accelerated.

Although run $\mathrm{C} 2$ satisfies the modified trapping condition of Equation (2), the acceleration efficiency, $N_{\mathrm{e}, \mathrm{BI}} / N_{\mathrm{e}, \text { tot }} \simeq 0.6$, is much less than that for run G2. In Figure 6 the spectrum for run $\mathrm{C} 2$ is also compared with the spectrum calculated for run G2 at a different phase of the shock reformation (denoted as run $\mathrm{G} 2^{*}$ ), at which the strength of the Buneman waves matches that for run $\mathrm{C} 2$. Still, the fraction of pre-accelerated electrons in run $\mathrm{G} 2 *$ is four times that in run $\mathrm{C} 2$, but the maximum energies of the electrons are comparable, $\max (\gamma) \approx 3-4$. It is clear that the Buneman wave strength is not the only parameter that determines the efficiency of SSA in the shock foot.

SSA consists of two individual processes: (1) interaction with electrostatic waves and (2) magnetic gyration. In the Appendix we present a detailed analytical treatment of the equation of motion of electrons in the wave field, demonstrating that the electrostatic field of the waves does the physical work. Here, we summarize the conclusions.

Figure 7 illustrates the first stage of the SSA process for the in-plane (left panels (a1)-(e1)) and the out-of-plane cases (right panels (a2)-(e2)). For specific electrons extracted from runs E2 and G2, we see the time evolution of the energy (Figure 7(b)) and the momentum (Figure 7(c)), as well as the electric field at the location of the particle in the simulation frame and in the instantaneous particle rest frame (Figures $7(\mathrm{~d})$ and (e), respectively). The latter is particularly interesting, because in the electron rest frame the electric field is the sole provider of acceleration. We refer to the selected electron in the in-plane case (left panels) as the first electron and the other one as the second electron. Initially both electrons move with the plasma bulk. To be trapped by electrostatic waves, the electrons must travel with the waves against the upstream plasma flow, and hence be picked up from the thermal pool. Before doing so, the electrons move in the negative $x$-direction undisturbed through several electrostatic wavefronts. Significant energy gain commences at time $t \omega_{\mathrm{pe}}=6270$ for the first electron and at $t \omega_{\mathrm{pe}}=3745$ for the second electron. The particles then remain trapped by the waves and undergo the first stage of acceleration at time intervals $t \omega_{\mathrm{pe}}=(6275-6300)$ for the first electron and $t \omega_{\mathrm{pe}}=(3745-3753)$ for the second electron. During this stage both electrons move in the direction of shock propagation, and their $p_{y}$ momentum remains small. The end of the first-stage acceleration is marked by the black vertical line in Figure 7 , beyond which the electrons resume gyrating.

The acceleration of the first electron occurs in the same way as in 1D geometry (Hoshino \& Shimada 2002): the electron is pushed toward the upstream region by the electrostatic field of a Buneman wave, which for some time compensates the $x$-component of the Larmor acceleration and thus keeps the electron roughly in phase with the wave. Consequently, the average values of $E_{x}$ and $E_{y}$ electric-field components are close to zero in the particle reference frame (Figure $7(\mathrm{e} 1)$ ). The continuous gradient in $p_{z}$ at $t \omega_{\mathrm{pe}}=(6275-6300)$ reflects the transverse Larmor acceleration, which can be described as the effect of the motional electric field in the frame of the electrostatic wave. It is important to note that for the in-plane magnetic field the wavefronts are infinitely extended in the $z$-direction, and the energy gain terminates when the electron loses phase coherence with the Buneman wave. In reality the energization will terminate earlier. In the upstream flow frame all the energy gain comes from the field of the Buneman wave though.

The second electron displays a similar behavior, but in the frame of the obliquely propagating waves. At $t \omega_{\mathrm{pe}} \simeq 3745$ it starts moving in the $x$-direction, but the electrostatic field of the waves roughly compensates the Larmor acceleration in the $y$-direction, as $E_{\mathrm{PRF}, \mathrm{y}} \approx 0$ (Figure $7(\mathrm{e} 2)$ ). Instead, the electron is accelerated in the $x$-direction by the electrostatic field of the Buneman waves. We conclude that in all cases the energy gain arises from the electrostatic field of the waves, while the formal acceleration reflects the competition of Larmor acceleration and that imposed by the waves. The in-plane configuration captures only part of the Buneman waves, as only wavevectors in the simulation plane are allowed, so there is a lower rate of energy gain compared to the out-of-plane case. In addition, the restriction of the wave phase velocity to the simulation plane changes the direction of sliding along a wavefront from effectively the $x$-direction to the $z$-direction.

Let us estimate the energy gain arising from trapping at an electrostatic wavefront. Equations (11) and (14) give the rate of energy gain for the out-of-plane and in-plane configurations, respectively. The phase speed that the electrons need to match is $v_{\mathrm{ph}, 0}=0.1 c$ and $v_{\mathrm{ph}, 90}=0.4 c$ for the in-plane and out-ofplane configurations, respectively, so $\Delta v$ and hence the energization rate is two times larger in the out-of-plane case than it is for in-plane magnetic field. The total energy gain is the product of the rate of gain and the time of interaction. The time of interaction is limited by three factors: the intermittency 

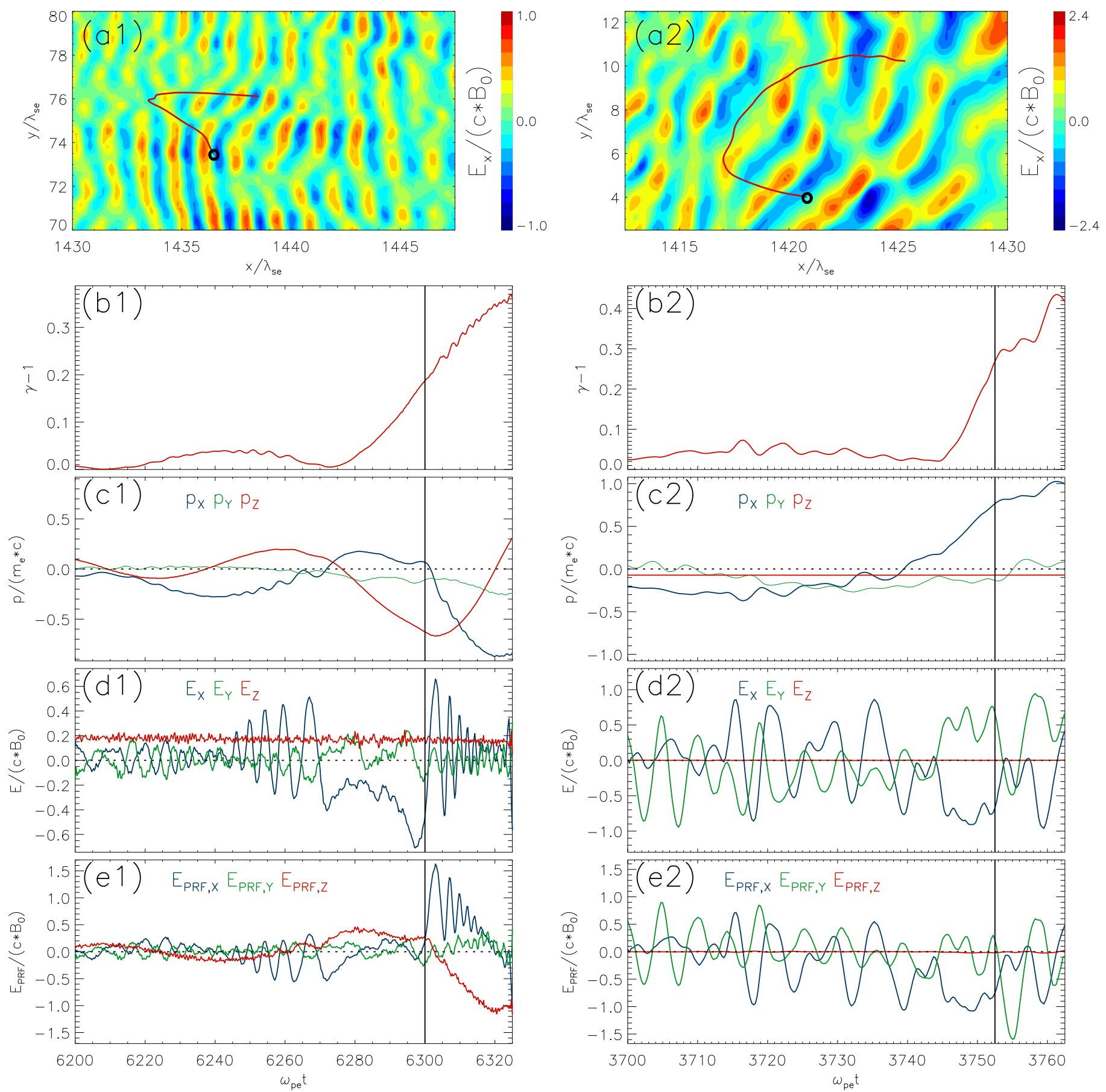

Figure 7. Interaction of electrons with Buneman waves for in-plane runs (panels (a1)-(e1), case E2) and out-of-plane runs (panels (a2)-(e2), case G2). Panels (a*): map of $E_{x}$ at the time indicated by the vertical black lines in the lower panels. Overlaid are the position of an electron (black dot) at the same time moment as $E_{x}$ maps, its trajectory history for the past $60 \omega_{\mathrm{ce}}^{-1}$, and past positions of the electron for every $\omega_{\mathrm{pe}} t=10$ intervals, designated with red dots. Panels (b*): evolution of electron energy. Panels $\left(\mathrm{c}^{*}\right)$ : evolution of electron momentum. Panels $\left(\mathrm{d}^{*}\right)$ : dimensionless components of electric field at electron position in the simulation frame. Panels $\left(\mathrm{e}^{*}\right)$ : components of electric field at electron position in the electron rest frame.

of waves, escape by acceleration perpendicular to the wavefront, and escape to the side of the wavefront.

In the in-plane case the wavefront is infinitely extended in the $z$-direction, and no escape to that side is possible. For an out-of-plane magnetic field and an average speed along the wavefront of $\sim(0.1-0.2) c$, the electrons would escape trapping on $t_{\mathrm{esc}} \approx(25-50) \omega_{\mathrm{pe}}^{-1}$, as the wavefronts in Figure 7 have a lateral extent of about $5 \lambda_{\mathrm{se}}$.
The escape time perpendicular to the wavefront can be estimated as $t_{\mathrm{esc}} \approx \pi \omega_{\mathrm{pe}}^{-1}\left(v_{\Phi}+v_{0}\right) / v_{\mathrm{e}, \mathrm{WRF}}$, where $v_{\mathrm{e}, \mathrm{WRF}}$ is the velocity of electrons in the wave frame. For the out-of-plane case this gives $t_{\mathrm{esc}, 90} \gtrsim 18 \omega_{\mathrm{pe}}^{-1}$, as the average electron speed $v_{\mathrm{e}, \mathrm{WRF}} \lesssim 0.1 c$.

The trapping time coming from the wave time intermittency can be estimated directly from simulations. The ability to accelerate an electron up to a certain energy depends not only 


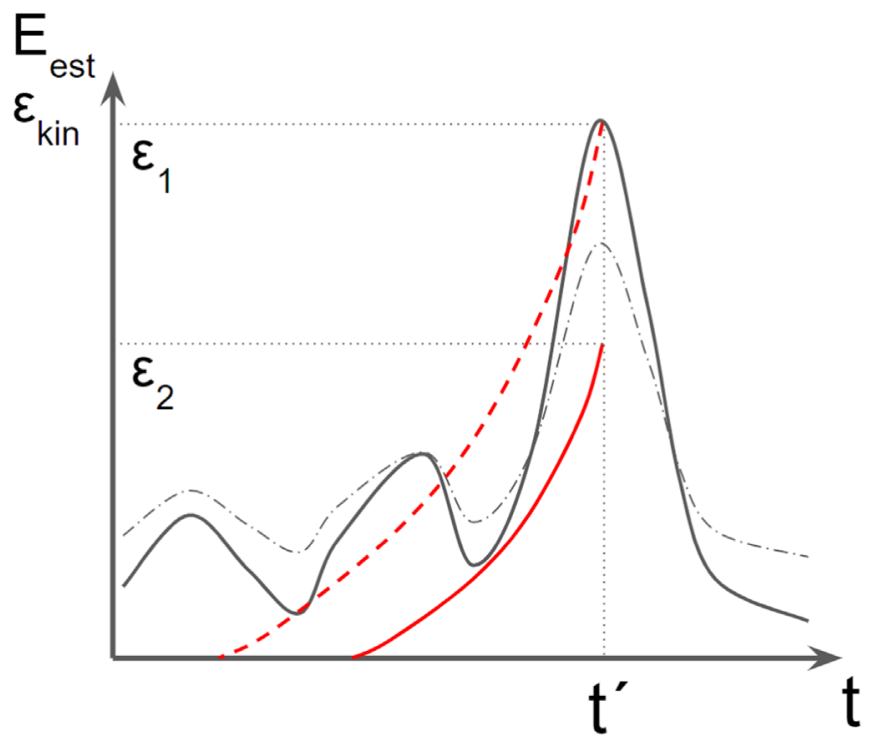

Figure 8. Schematic time evolution of the electrostatic field strength (black dashed-dotted line) at a chosen location in the Buneman wave rest frame. The black solid line is the maximal energy of the electron that can be trapped by the electrostatic field. The red lines represent the energy histories of electrons, for which trapping is possible (red solid line) and impossible (red dashed line).

on the instantaneous local electrostatic field strength but also on the previous strength history and the ability to trap an electron during the whole acceleration period. In Figure 8 the time evolution of the electrostatic field strength $\left(E_{\mathrm{ES}}\right.$, black dasheddotted line) at a chosen location is presented. This field is able to trap the electrons with energies (shown by the black solid line), which is calculated assuming the equality between electrostatic and Lorentz forces at the chosen location. At a time $t^{\prime}$ the electrostatic field can trap an electron with energy $\epsilon_{1}$. However, taking into account the evolution of $E_{\mathrm{ES}}$ and the energy of electrons (red dashed line) this electron cannot be trapped during the whole acceleration period. Therefore, the electrons with a final energy $\epsilon_{2}$ and their energy history shown with the red solid line can be present in the simulation. According to these considerations the trapping time reads

$$
t_{\mathrm{tr}, 0} \approx 13 \omega_{\mathrm{pe}}^{-1} \text { and } t_{\mathrm{tr}, 90} \approx 11 \omega_{\mathrm{pe}}^{-1},
$$

which is approximately the time that we analytically estimated based on the acceleration in the direction of the wave motion (see the Appendix). Thus, one of the main limiting factors for electron acceleration is the intermittency of the Buneman waves.

Calculated average energy gains are

$$
\Delta \gamma_{0} \approx 0.18 \text { and } \Delta \gamma_{90} \approx 0.42
$$

which are similar to those for electrons in Figure 7 and average energies of accelerated electrons in Figures 5 and 6. The analytically expected energy increase can be written as

$$
\begin{aligned}
\Delta \varepsilon & \approx e E_{\mathrm{ES}}|F| \Delta v t_{\mathrm{tr}} \\
& =\Delta v^{2} t_{\mathrm{tr}} \omega_{\mathrm{pe}} m_{\mathrm{e}}\left(m_{\mathrm{e}} / m_{\mathrm{i}}\right)^{(1 / 6),}
\end{aligned}
$$

where $e E_{\mathrm{ES}}=m_{\mathrm{e}} \Delta v \omega_{\mathrm{pe}}\left(m_{\mathrm{e}} / m_{\mathrm{i}}\right)^{(1 / 6)} \quad$ (Ishihara et al. 1980; Amano \& Hoshino 2009b; Matsumoto et al. 2012) and $|F|$ is assumed to be about 1 . Therefore, the main difference in the acceleration rate comes from velocity difference, $\Delta v=\left(v_{\Phi}+v_{0}\right)$, and the energy gain of electrons is still stronger in the out-of-plane case due to a higher phase speed of the Buneman waves.

We note that the modified trapping conditions (Equation (2) or (3)) refer to reaching a certain strength of the electrostatic field that is needed for trapping, while the energy gain of electrons is related to the velocity difference between reflected ions and upcoming electrons. This velocity difference imposes the main restriction for the in-plane simulations in their applicability to mimic realistic SSA efficiency. Using a higher Mach number cannot significantly change the SSA efficiency in case of the same velocity difference defined by the magneticfield configuration. For the same mass ratio the number of preaccelerated electrons is larger by about $(30-40) \%$ in the runs with a higher Alfvénic Mach number (see Table 2, runs B2-C2 and D2-E2), which is not the factor of 10 required to reach the SSA efficiency seen in out-of-plain runs. Therefore, significant modifications of the parameters of the simulation (not just a change of the Alfvénic Mach number) are needed to reproduce the out-of-plain SSA efficiency by means of in-plane simulations.

The energy difference associated with climbing or sliding down the potential well of a Buneman wave can be estimated as $\Delta \gamma m c^{2}=e E_{\mathrm{BI}} \lambda_{\mathrm{BI}} / 2 \pi$. The wavelength of Buneman waves, $\lambda_{\mathrm{BI}}=2 \pi \Delta v / \omega_{\text {pe }}$, then implies an energy change $\Delta \gamma \approx 0.05$ in the in-plane case and $\Delta \gamma \approx 0.17$ in the out-of-plane run. This is insufficient to redirect an incoming electron to stationarity in the wave frame. Fluctuations in the Buneman wave field are clearly needed to trap particles and keep them in phase with the waves.

We observe that for $\varphi=90^{\circ}$ larger numbers of electrons are picked up from the bulk plasma for further acceleration than is seen with the in-plane configuration, which can be explained by a twice stronger $e E_{\mathrm{ES}}$ force in the out-of-plane case.

\section{Summary and Discussion}

We analyze electron injection processes at nonrelativistic perpendicular collisionless shocks with high Alfvénic Mach numbers with 2D3V numerical PIC simulations. Earlier studies indicated that SSA operates at the leading edge of the foot as a first-stage electron pre-acceleration mechanism, provided the Alfvénic Mach number satisfies a condition of efficient driving of the electrostatic Buneman waves (the trapping condition, Matsumoto et al. 2012). In Matsumoto et al. (2015) and Bohdan et al. (2017) we showed that in 2D simulations that use a field component that lies in the simulation plane, the downstream nonthermal-electron fraction is much lower than that with an out-of-plane mean field. Noting that much of this difference results from an incomplete account of the Buneman instability in the in-plane geometry, and motivated by results of recent $3 \mathrm{D}$ studies that demonstrate that the injection physics past the SSA stage can adequately be studied with 2D in-plane simulations (Matsumoto et al. 2017), here we further investigate electron acceleration by SSA at perpendicular high- $M_{\mathrm{A}}$ shocks with in-plane magnetic-field configurations. The aim is to infer the SSA efficiency, in particular the validity of the trapping condition in its original form and the variant proposed in Bohdan et al. (2017), and the relation to the SSA efficiency observed in simulations with the out-of-plane fields.

Our results can be summarized as follows:

1. The energy gain in SSA always arises from the electrostatic field of a Buneman wave with which the 
electron travels for some time. The apparent acceleration, $\dot{\boldsymbol{v}}$, reflects the superposition of electrostatic acceleration and Larmor acceleration that might be described as effect of the motional electric field in the wave frame. This process is more efficient in the out-of-plane case because both the phase speed and the amplitude of the waves are higher than for $\varphi=0^{\circ}$.

2. As in high- $M_{\mathrm{A}}$ shock simulations with out-of-plane magnetic fields, for in-plane magnetic field the strength of the electrostatic wave modes in the shock foot is determined by the Alfvénic Mach number in relation to the trapping condition. The more $M_{\mathrm{A}}$ exceeds the trapping condition, the stronger the intensity of the Buneman waves. Shocks with Alfvénic Mach numbers satisfying the trapping condition by a similar margin show comparable wave strengths in simulations for different ion-to-electron mass ratios.

3. Shocks in simulations with an in-plane magnetic field demonstrate electrostatic wave intensities lower than those observed in the out-of-plane case, even if the modified trapping condition is satisfied.

4. The trapping time is mostly defined by intermittency of, and limited phase coherence of electrons with, the Buneman waves. This limits the duration of the velocity match between electrons and the waves.

5. The number of electrons pre-accelerated via SSA in the shock foot strongly correlates with the strength of the electrostatic waves. Shocks with the same physical conditions defined through the trapping condition show similar SSA efficiency. The latter is proportional to $M_{\mathrm{A}}$ for a given mass ratio. However, SSA always produces larger fractions of pre-accelerated electrons in simulations with the out-of-plane configurations, even if the intensities of the Buneman waves are similar as in the in-plane case. One reason for that is the larger number of electrons being picked up from the bulk plasma for SSA compared to the in-plane configuration.

We conclude that with an in-plane magnetic-field configuration we cannot achieve the same level of SSA efficiency as in simulations with out-of-plane magnetic field or 3D simulations (Matsumoto et al. 2017), unless the parameters and settings of the simulation setup are significantly modified.

This paper is conceived as the first of a series investigating different aspects of electron acceleration processes at nonrelativistic perpendicular shocks using PIC simulations. Interaction with Weibel filaments and magnetic reconnection in the shock transition, plasma heating, and the generation of turbulent magnetic field will be covered in forthcoming publications.

We thank the anonymous referee for their comments. The work of J.N. has been supported by Narodowe Centrum Nauki through research project DEC-2013/10/E/ST9/00662. This work was supported by JSPS-PAN Bilateral Joint Research Project grant No. 180500000671. The numerical experiment was possible through a 10 Mcore-hour allocation on the 2.399 PFlop Prometheus system at ACC Cyfronet AGH. Part of the numerical work was conducted on resources provided by the North-German Supercomputing Alliance (HLRN) under projects bbp00003 and bbp00014.

\section{Appendix Analytical Model of Electron SSA}

\section{A1. Out-of-plane Configuration, $\varphi=90^{\circ}$}

In the simulation frame, the large-scale magnetic field of the right plasma slab, $\boldsymbol{B}=B_{0} \hat{z}$, induces a motional electric field, $\boldsymbol{E}=-v_{0} B_{0} \hat{\boldsymbol{y}}$, where $v_{0}$ is the speed of the upstream plasma flowing in the $-\mathrm{x}$-direction. The entire Larmor orbit of all particles with low temperature is leveled in the simulation plane, as are the acceleration imposed by the waves.

Suppose an electrostatic wave propagates at an angle $\Theta$ to the $x$-axis. The electric field carried by the wave is

$$
E_{x}=E_{\mathrm{ES}} F \cos \Theta \quad E_{y}=E_{\mathrm{ES}} F \sin \Theta,
$$

where the wave factor is

$$
F=\sin \left(\frac{\omega_{\mathrm{pe}}}{v_{\Phi}+v_{0}}\left[x \cos \Theta+y \sin \Theta-v_{\Phi} t\right]+\Phi\right) .
$$

Here we allow for an arbitrary phase, $\Phi$. The phase speed of the wave, $v_{\Phi}$, is measured in the simulation frame. The wave number is related to the velocity of reflected ions through the resonance condition of the Buneman modes, $\omega_{\mathrm{pe}}=k\left(v_{\Phi}+v_{0}\right)$.

Now consider an electron with velocity components $v_{x}$ and $v_{y}$. Using nonrelativistic kinematics we find the acceleration of the electron as

$$
\begin{aligned}
& \dot{v}_{x}=-\Omega_{\mathrm{e}} \frac{E_{\mathrm{ES}}}{B_{0}} F \cos \Theta-\Omega_{\mathrm{e}} v_{y} \\
& \dot{v}_{y}=\Omega_{\mathrm{e}} v_{0}+\Omega_{\mathrm{e}} v_{x}-\Omega_{\mathrm{e}} \frac{E_{\mathrm{ES}}}{B_{0}} F \sin \Theta .
\end{aligned}
$$

Let us rotate the coordinate system by an angle $\Theta$, so that $x^{\prime}$ is oriented in the direction of motion of the waves and $y^{\prime}$ is perpendicular to it. The corresponding accelerations then read

$$
\begin{aligned}
& \dot{v}_{x^{\prime}}=\Omega_{\mathrm{e}}\left(v_{0} \sin \Theta-\frac{E_{\mathrm{ES}}}{B_{0}} F-v_{y^{\prime}}\right) \\
& \dot{v}_{y^{\prime}}=\Omega_{\mathrm{e}}\left(v_{0} \cos \Theta+v_{x^{\prime}}\right) .
\end{aligned}
$$

The wave factor, $F$, is explicitly time-dependent and may induce rapidly oscillating acceleration. The other terms only describe Larmor gyration in the flow frame and hence no real energy gain. The wave factor must be approximately constant, if continuous energy gain is to be achieved for about 10 plasma times, $\omega_{\text {pe }}^{-1}$, as observed. This requires that on average $v_{x^{\prime}}-v_{\Phi} \lesssim 0.2 c$ or roughly acceleration from $v_{x^{\prime}}=0.2 c$ to $v_{x^{\prime}}=0.6 c$, after which the electron is out of phase with the wave and commences Larmor motion.

The Larmor motion of the reflected ions mandates a wave direction for which $\sin \Theta$ is negative. Likewise, the wave factor, $F$, must be negative to effect energy gain. Equation (10) then indicates that acceleration in $y^{\prime}$ direction follows that in $x^{\prime}$ direction, and for a fair range of initial conditions $\dot{v}_{y^{\prime}}$ is slightly less than $\dot{v}_{x^{\prime}}$ and increases with the same rate, at least for up to $1 \Omega_{\mathrm{e}}^{-1} \simeq 12 \omega_{\mathrm{pe}}^{-1}$. Correspondingly, the momentum component $p_{x}$ increases approximately linearly, and the increase in speed is approximately $E_{\mathrm{ES}} /\left(2 B_{0}\right)$, whereas $p_{y}$ remains approximately constant.

The effective acceleration toward the upstream region arises from the superposition of acceleration in the electrostatic field of the Buneman waves and the Larmor acceleration, whicj are oppositely directed in the $y$-direction, but both have positive 
components in the x-direction. Acknowledging that both $F$ and $\sin \Theta$ must be negative, the rate of energy gain in the upstream flow frame is

$$
\begin{aligned}
& m \frac{d}{d t} \frac{\left(v_{x}+v_{0}\right)^{2}+v_{y}^{2}}{2} \\
& \quad=e E_{\mathrm{ES}}|F|\left[\left(v_{x}+v_{0}\right) \cos \Theta-v_{y}|\sin \Theta|\right]
\end{aligned}
$$

and hence completely independent of the motional electric field. In the simulation frame the velocity component $v_{0} \cos \Theta$ disappears from Equation (11) and a new component of energy-gain rate appears, $\Omega_{\mathrm{e}} v_{0} v_{y}$, which captures the apparent energy by Larmor motion in this frame.

\section{A2. In-plane Configuration, $\varphi=0^{\circ}$}

The main impact of the in-plane configuration is that part of the Larmor motion is perpendicular to the simulation plane, so the orientation and properties of the Buneman waves are modified, as only wavevectors in the simulation plane can be captured. The wave factor changes to

$$
F=\sin \left(\frac{\omega_{\mathrm{pe}}}{v_{\Phi}+v_{0}}\left[x-v_{\Phi} t\right]+\Phi\right) .
$$

The acceleration then follows by appropriate rotation of that given in Equation (9),

$$
\begin{aligned}
& \dot{v}_{x}=-\Omega_{\mathrm{e}} \frac{E_{\mathrm{ES}}}{B_{0}} F+\Omega_{\mathrm{e}} v_{z} \\
& \dot{v}_{z}=-\Omega_{\mathrm{e}} v_{0}-\Omega_{\mathrm{e}} v_{x} .
\end{aligned}
$$

Obviously, there is linear acceleration in the -z-direction, if the particle can be held at an approximately constant phase $(F<0$; $v_{x} \approx v_{\Phi}$ ) in the wave. As $v_{\Phi} \gtrsim v_{0}$ it is the Larmor acceleration that is responsible for the particle's sliding along the wavefront, and the electrostatic field of the waves provides slow energy gain at a rate

$$
m \frac{d}{d t} \frac{\left(v_{x}+v_{0}\right)^{2}+v_{z}^{2}}{2}=e E_{\mathrm{ES}}|F|\left(v_{x}+v_{0}\right),
$$

which also only involves the electrostatic field of the Buneman waves. The energy gain will be less than that for the out-of-plane configuration, because only part of the motion of the backstreaming ions can drive waves that hence have lower amplitude, $E_{\mathrm{ES}}$, and additionally the velocity term in Equation (14) is reduced.

\section{ORCID iDs}

Artem Bohdan 나 https://orcid.org/0000-0002-5680-0766 Jacek Niemiec (i) https://orcid.org/0000-0001-6036-8569 Martin Pohl (1) https://orcid.org/0000-0001-7861-1707 Masahiro Hoshino (i) https://orcid.org/0000-0002-1818-9927

\section{References}

Aharonian, F. A. 2013, APh, 43, 71

Amano, T., \& Hoshino, M. 2009a, ApJ, 690, 244

Amano, T., \& Hoshino, M. 2009b, PhPl, 16, 102901

Axford, W. I., Leer, E., \& Skadron, G. 1977, Proc. ICRC, 11, 132

Blandford, R., \& Eichler, D. 1987, PhR, 154, 1

Bocchino, F., Orlando, S., Miceli, M., \& Petruk, O. 2011, A\&A, 531, A129

Bohdan, A., Niemiec, J., Kobzar, O., \& Pohl, M. 2017, ApJ, 847, 71

Buneman, O. 1958, PhRvL, 1, 8

Buneman, O. 1993, in Computer Space Plasma Physics: Simulation Techniques and Software, ed. H. Matsumoto \& Y. Omura (Tokyo: Terra), 67, https://www.terrapub.co.jp/e-library/cspp/

Drury, L. O. 1983, RPPh, 46, 973

Hoshino, M., \& Shimada, N. 2002, ApJ, 572, 880

Ishihara, O., Hirose, A., \& Langdon, A. B. 1980, PhRvL, 44, 1404

Kato, T. N., \& Takabe, H. 2010, ApJ, 721, 828

Matsumoto, Y., Amano, T., \& Hoshino, M. 2012, ApJ, 755, 109

Matsumoto, Y., Amano, T., \& Hoshino, M. 2013, PhRvL, 111, 215003

Matsumoto, Y., Amano, T., Kato, T. N., \& Hoshino, M. 2015, Sci, 347, 974

Matsumoto, Y., Amano, T., Kato, T. N., \& Hoshino, M. 2017, PhRvL, 119, 105101

Niemiec, J., Pohl, M., Bret, A., \& Wieland, V. 2012, ApJ, 759, 73

Niemiec, J., Pohl, M., Stroman, T., \& Nishikawa, K.-I. 2008, ApJ, 684, 1174

Petruk, O., Dubner, G., Castelletti, G., et al. 2009, MNRAS, 393, 1034

Rothenflug, R., Ballet, J., Dubner, G., et al. 2004, A\&A, 425, 121

Schneiter, E. M., Velázquez, P. F., Reynoso, E. M., \& de Colle, F. 2010, MNRAS, 408, 430

Schneiter, E. M., Velázquez, P. F., Reynoso, E. M., Esquivel, A., \& De Colle, F. 2015, MNRAS, 449, 88

Stroman, W., \& Pohl, M. 2009, ApJ, 696, 1864

West, J. L., Safi-Harb, S., Jaffe, T., et al. 2016, A\&A, 587, A148

Wieland, V., Pohl, M., Niemiec, J., Rafighi, I., \& Nishikawa, K.-I. 2016, ApJ, 820,62 